\title{
Comparison of Maternal and Fetal Outcomes in Pregnancies with Preterm Premature Rupture of Membrane (PPROM) Terminating in 34th or 36th Gestational Weeks: A Clinical Trial
}

Shamsi Abbasalizadeh ${ }^{1}$, Rana Bagherifard ${ }^{1 *}$, Farshad Mahdavi ${ }^{2}$, Fatemeh Abbasaizadeh ${ }^{1}$, Shiva Raouf ${ }^{3}$

1. Department of Gynecology, Faculty of Medicine, Tabriz University of Medical Science, Tabriz, Iran

2. Department of Surgery, Faculty of medicine, Tabriz University of Medical Science, Tabriz, Iran

3. Women's Reproductive Health Research Center .Tabriz University of Medical Science ,Tabriz ,Iran

\begin{abstract}
Introduction: PPROM, rupture of amniotic sac prior to 37th gestational week and before delivery, can lead to maternal complications such as delivery complications, emergency cesarean section, chorioamnionitis, endometritis, sepsis and maternal death. Neonatal complications also include preterm delivery, placental abruption, umbilical cord prolapse, NICU admission, respiratory distress syndrome and neonatal necrotizing enterocolitis. In the present study, we aimed at studying maternal and neonatal outcomes in patients with terminated pregnancy in 34th and 36th gestational weeks. Materials and methods: 40 pregnant women, with PPROM who underwent pregnancy termination at 34 group $(A)$ or 36 group (B) gestational weeks, were included to be evaluated and compared for maternal and neonatal outcomes. Type of delivery, birth complications, chorioamnoionitis, endometritis, sepsis, maternal mortality, infant gender, birth weight, Apgar scores, respiratory distress syndrome, Meconium-stained amniotic fluid, NICU admission, abruption, umbilical cord prolapse, maternal and neonatal outcomes were compared between the two groups. Results: There was no statistically significant difference between the two groups regarding maternal age, level of education, or gravity. The percentage of cases with birth weight between 1500 and $2500 \mathrm{~g}$ was significantly higher in group $\mathrm{A} P<0.001)$. Frequency of NICU admission in group A was significantly more than group $B(P<0.001)$. In conclusion: Termination of pregnancy at 36 weeks compared to 34 weeks in pregnant women with PPROM is preferred in terms of neonatal outcomes and it is recommended; also, there might be no preference in terms of maternal outcomes.
\end{abstract}

Key words: Preterm Birth; Preterm Prelabour rupture of membranes; chorioamnoionitis; infantal respiratory distress syndrome;

\section{Corresponding author:}

Rana Bagherifard

Department of Gynecology, Faculty of Medicine, Tabriz University of Medical Science, Tabriz, Iran

Email:Shamciabbasalizadeh@gmail.com

Receive date: 2017-01-24 | Accept date: 2017-03-26 | Publish date: 2017-04-08

DOI: $10.7575 /$ aiac.abcmed.17.05.02.02 


\section{Introduction}

PPROM has been defined as rupture of amniotic sac prior to 37th gestational week and before delivery (1-5). Althogh the main underlying cause of PPROM is not well understood (6-10), early dilation of the cervix, uterus or fetus infections, and premature delivery has been suggeted to predispose PPROM (11-15). Based on its pathophysiology, PPROM may lead to maternal and neonatal complications (15-20). Considering maternal outcomes, PPROM may cause delivery complications, emergency cesarean section, chorioamnionitis, endometritis, sepsis and maternal death in more severe cases (25-30). However, neonatal complications include preterm delivery, placental abruption, umbilical cord prolapse, NICU admission, respiratory distress syndrome and neonatal necrotizing enterocolitis (30-36). Dealing with women suffering PPROM is still controversial. Some studies recommended "wait and watch" management to be supperior to early termination of pregnancy (9-11). However, these studies have not focused on comparison of pregnancy outcomes at 34th and 36th gestational weeks (12). In another study by Kayem et al., the maternal and neonatal outcomes of labor induction and wait and watch management in women with PPROM at 34th and 36th gestational weeks were studied retrospectively. Accordingly, induction of labor at 34th week increased newborn morbidity; while, wait and watch management increased risk of infection (chorioamnionitis) (13). Buchanan et al. showed that the methodology of existing studies is not comprehensive; thus, it no definitive conclusions can be proposed for the management of PPROM (9). In the present study, we aimed at studying maternal and neonatal outcomes in patients with terminated pregnancy in 34th and 36th gestational weeks.

\section{Materials and methods}

Patients

40 pregnant women, with PPROM who underwent pregnancy termination at 34 group (A) or 36 group (B) gestational weeks, were included to be evaluated and compared for maternal and neonatal outcomes. Patients were randomly divided into two groups using online randomization (www.randomized.com). All subjects signed a written informed consent. This study was approved by the Ethics Committee of Tabriz University of Medical Sciences. This study was registered on the Clinical trials site by the number of IRCT2014120120168N1. All patients with approved PPROM that underwent termination of pregnancy at 34 or 36 gestational weeks were included. Exclusion criteria were as follows: underlying diseases such as diabetes and hypertension, other pregnancy-related diseases such as pre-eclampsia and eclampsia, PPROM terminated in the other gestational weeks, major anomalies and life-threatening neonatal conditions, obstetric complications, history of previous infection, history of smoking or alcohol consumption. Later, type of delivery, birth complications, chorioamnoionitis, endometritis, sepsis, maternal mortality, infant gender, birth weight, Apgar scores, respiratory distress syndrome, Meconium-stained amniotic fluid, NICU admission, abruption, umbilical cord prolapse, maternal and neonatal outcomes were compared between the two groups. Maternal outcomes included complications associated with delivery, emergency cesarean section, chorioamnoionitis, endometritis, sepsis and maternal mortality. Neonatal outcomes included placental abruption, umbilical cord 


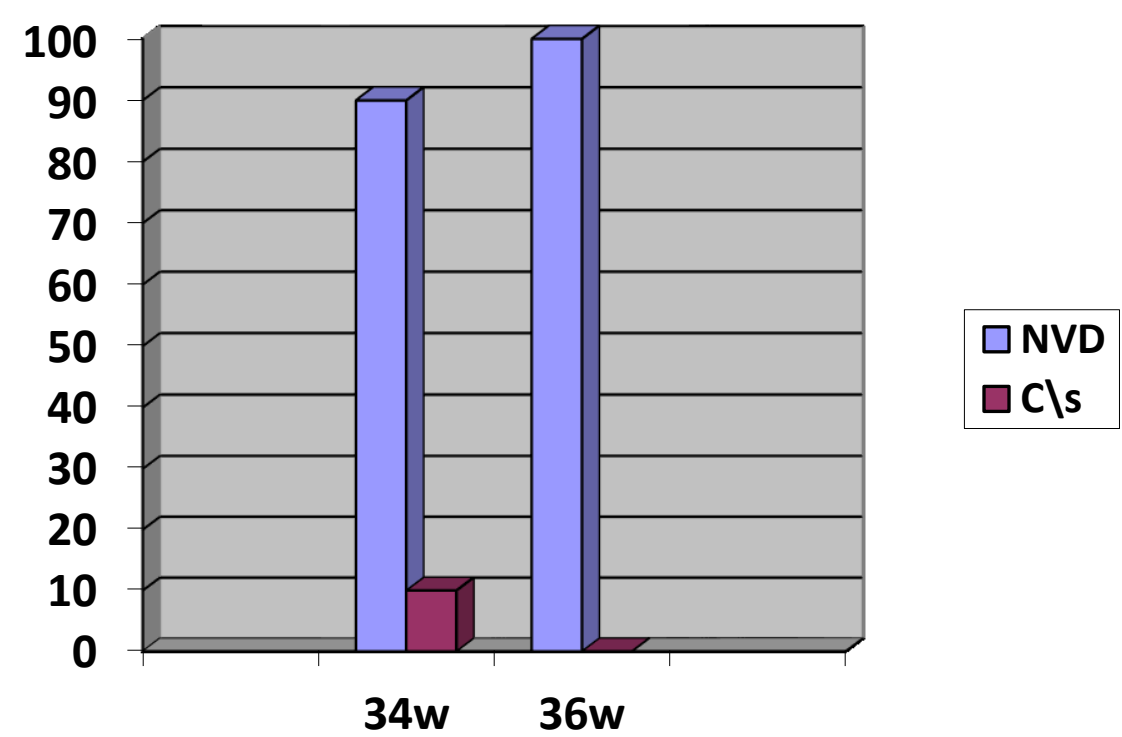

Figure 1: Frequency of delivery methods in the patients of both groups

prolapse, NICU admission, neonatal respiratory distress, necrotizing enter colitis and death of the fetus/newborn.

Statistical Analysis: Quantitative data were described by Mean $\pm S D$. The used statistical program was SPSS ${ }^{\mathrm{TM}}$ Version 21 . To compare quantitative data in independent groups, t-test was used. To compare qualitative data Chisquare test or Fisher's exact test was used. In all cases $\mathrm{P}<0.05$ was considered significant.

\section{Results}

Maternal age: Maternal age in the group $A$ was averagely $27.6 \pm 4.4$ years, and in group $B$ it was averagely $25.9 \pm 4.9$ years. There was no statistically significant difference between the two groups. Level of Education: Group of 34 week included illiterate in 3 cases (15\%), underDiploma in 14 (70\%), Diploma in 1 (1\%) and with university studies in $2(10 \%)$, respectively. Group B included illiterate in 6 cases (30\%), under-Diploma in 11 cases (55\%) and with university studies in 3 cases (15\%), respectively. There was no significant difference in both groups in this regard. Gravity: In group 34 week, gravid one was seen in 14 cases (70\%), gravid two in five (25\%) and gravid three in 1 case (5\%), and in the group B gravid one in 12 $(60 \%)$, gravid two in five (25\%) and gravid three in 3 cases (15\%) were reported respectively. Parity: In group A parity included null parity in 14 cases $(70 \%)$, Para One in five $(25 \%)$ and Para two in 1 case $(5 \%)$, and null parity in group $B$ included 12 patients (60\%), Para one in five (25\%) and Para-two in three (15\%), respectively. Abortion: Abortion was seen only in 1 case $(5 \%)$ in the group $A$. There were no significant differences between two groups in this regard. A history of preterm birth: There was no history of preterm birth in any group. Background of previous PPROM: In none of the groups history of previous PPROM existed. Previous delivery complications: There was no previous history of birth complications in any group. A history of previous infection: There was no history of previous infection in any group. History of smoking, alcohol: There was no history of tobacco or alcohol use in any group. Type of delivery: In group A, 18 cases with vaginal delivery and 2 cases with cesarean and in group B all cases had vaginal delivery. There was no significant difference between two groups in this regard (Figure 1). Delivery complications: There were no complications in delivery. Chorioamnionitis: Chorioamnionitis 


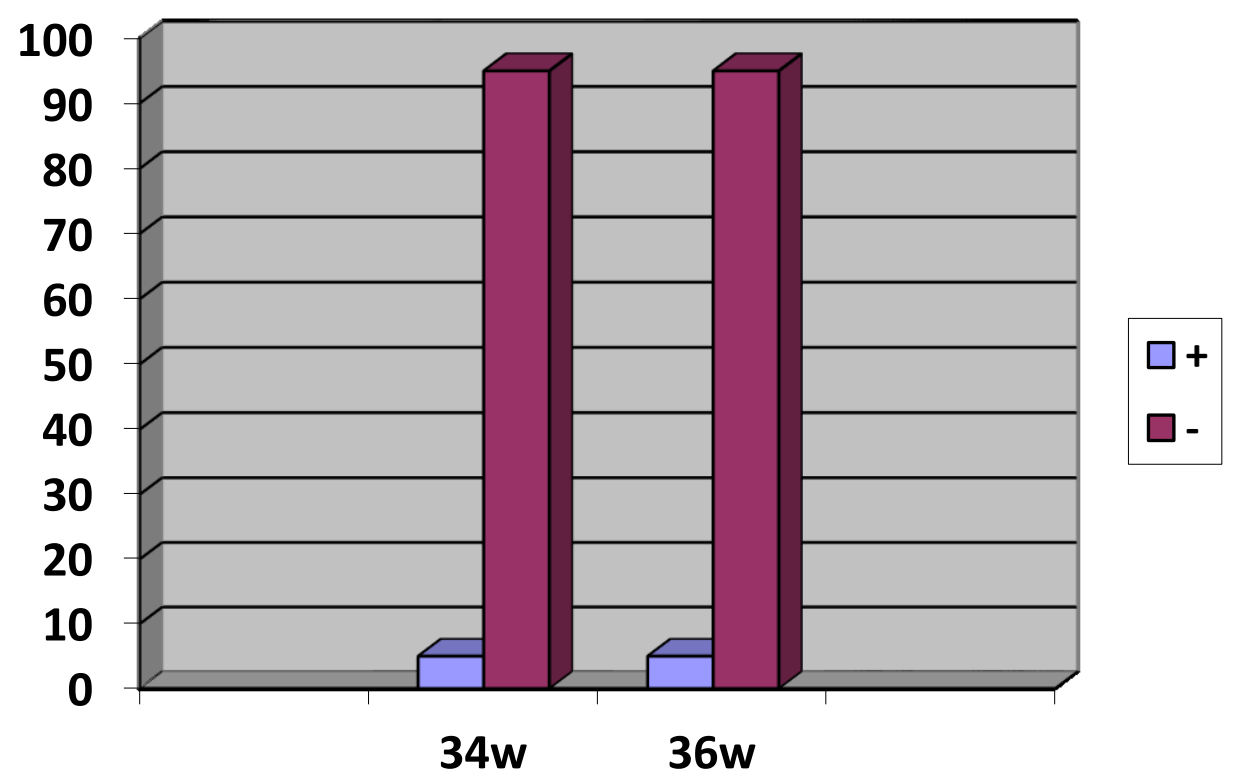

Figure 2: Frequency of Chorioamnionitis occurrence $(+)$ in the patients of both groups

was observed in 1 patient in both groups. There was no significant difference in this respect between the two groups (Figure 2). There was no endometritis in any group.

36w Birth weight in group $A$ in 18 newborns was between 1500 and $2500 \mathrm{~g}$, in one case it was between 2500 and $3500 \mathrm{~g}$, and in one case between 3500 and $4500 \mathrm{~g}$. In group B, birth weight of one case was 1,500 up to $2500 \mathrm{~g}$ and in 19 cases it was between 2500 and $3500 \mathrm{~g}$, respectively. There was no sepsis in any group. Maternal death: No maternal death was reported in any group. Newborn gender: In group A, 14 newborns (70\%) were male and 6 $(30 \%)$ were female and in group B, 9 newborns (45\%) were male and 11 newborns (55\%) were female. There was no significant difference between groups in this regard. Birth weight: The percentage of cases with birth weight between 1500 and 2500 g was significantly higher in group A $P<0.001$ ) (Figure 3 ). Fifth minute Apgar: In group A, fifth minute Apgar of newborns was between 3 and 5 in 1 case, between 5 to 7 in 6 cases and in 13 cases varied between 7 and 10 . In group $B$, the fifth minute Apgar of newborns varied between 3 to 5 in a case, between 5 to 7 in one case and varied between 7 and 10 in 18 cases. There was no significant difference between the frequency of the Apgar score of less than 7 and more than 7 in two groups (Figure 4). Neonatal respiratory distress syndrome was observed in 1 patient in both groups. There was no significant difference in this respect between two groups (Figure 5). Frequency of NICU admission in group $A$ was significantly more than group $B$ $(P<0.001) \quad$ (Figure 6). Meconium-stained amniotic fluid: Meconium-stained amniotic fluid was observed in group A in 2 cases (10\%), while it was not observed in group B. There was no significant difference between two groups. NICU admission: In group A, 13 newborns and in group B, 2 newborns were admitted in NICU. No case of umbilical cord detachment, umbilical cord prolapse, sepsis, NEC, or neonatal death were observed.

\section{Discussion}

Although PPROM is a known condition in obstetrics and gynecology, there are arguments on its correct management. One of the most important cases that has not been agreed upon is duration of the patient management in 


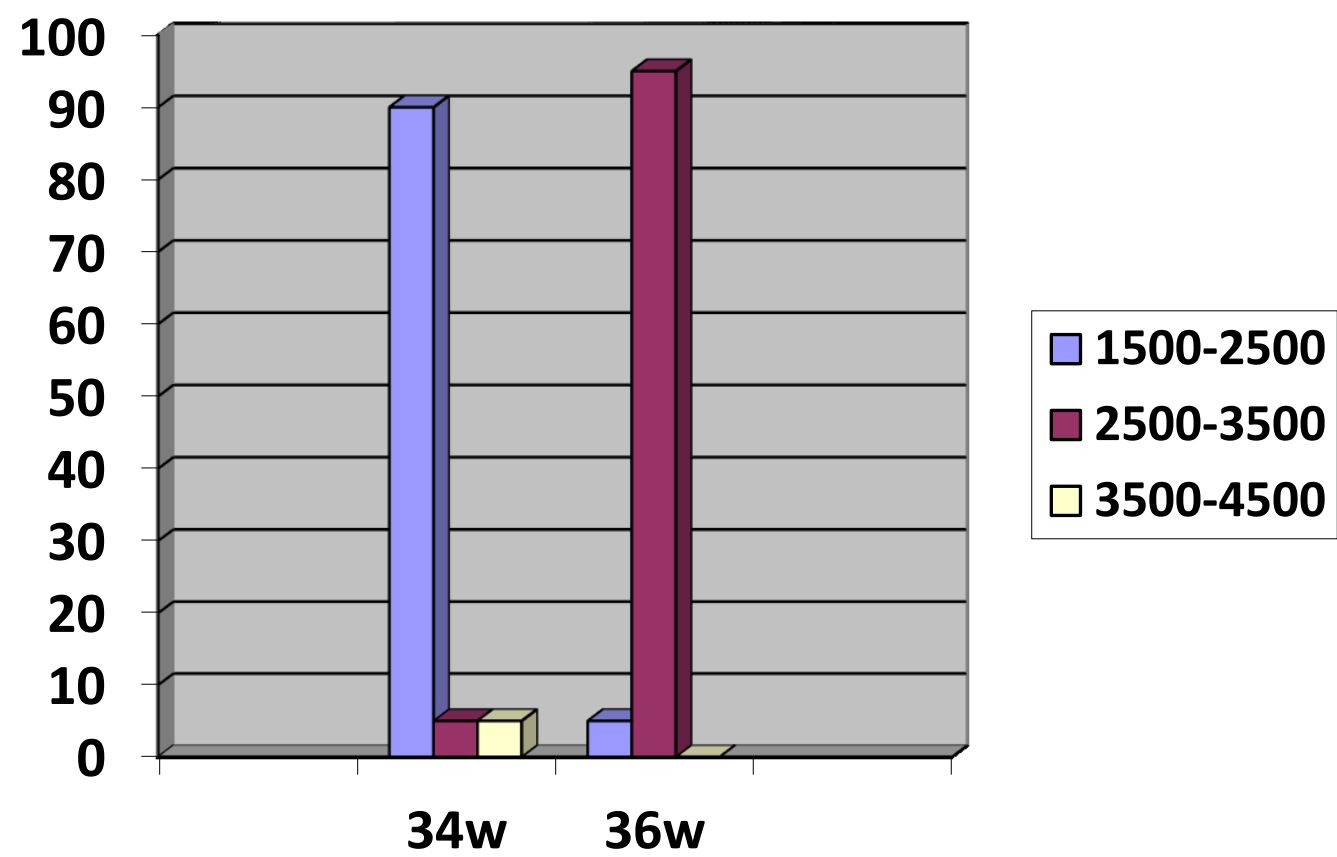

Figure 3: Frequency of newborns birth weight in both groups

pregnancies with PPROM $(4,8,25,31)$. In this study, maternal and neonatal outcomes in women with PPROM in particular periods of 34 and 36 gestational weeks were investigated. Accordingly, in the group with pregnancy terminated at 34 weeks compared to the group with pregnancy terminated at 36 weeks, birth weight was significantly lower and the frequency of NICU admission was high. However, other studied outcomes such as infection, type of delivery and respiratory distress syndrome were similar in both groups. The results of the studies conducted to compare induction of labor and expectant management of pregnant women with PPROM are variable and sometimes contradictory: In the findings of this study, Chorioamnionitis and mother average duration of hospital stay was significantly higher in the second group. Neonatal sepsis was more in this group, although the difference was not statistically significant. Finally, it was concluded that induction of labor during 34 and 36 gestational weeks was safe and prevent mother- fetus infectious complications (12). Study by Mercer et al. showed that induction of labor and expectant management during 32 to 36 gestational weeks resulted in pregnant women with PPROM were compared. 46 patients had undergone induction of labor and 47 patients had expectant management. Accordingly, the average of hospital stay for mother and newborn after birth was significantly higher in expectant management group. Chorioamnionitis and neonatal sepsis occurred more frequently in this group, although the differences were statistically not significant (28). The results of low frequency of Chorioamnionitis in two groups and lack of sepsis in the current study were similar to above research. Naef et al. compared the results of the labor induction during 34 and 37 gestational weeks and expectant management in PPROM. 57 patients in group one and 63 patients in group two were studied. They suggested that treatment with induction of labor reduced infection significantly which is in line with the results obtained from our study. In a comprehensive study by Hannah et al., induction of labor and expectant management 


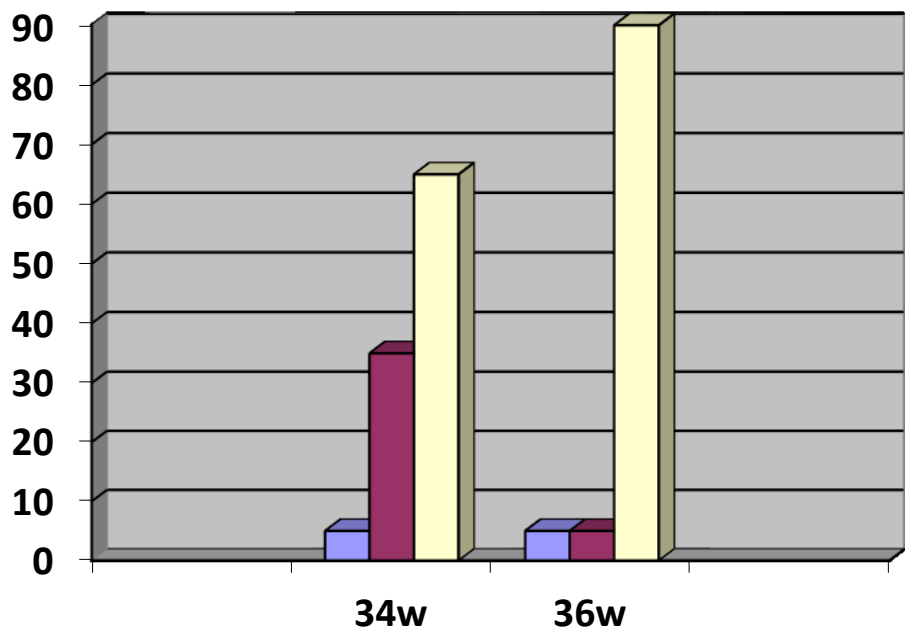

\section{$\square 3$ to 5 \\ $\square 5$ to 7 \\ $\square 7$ to 10}

Figure 4: Frequency of the neonatal Apgar in both groups

results in 5041 pregnant women with PPROM were compared. The results of this study showed that these two types of patient management had similar results on neonatal infection and need for cesarean section (32). We also did not find any difference between two groups 34 and 36 weeks on the incidence of neonatal infection and type of delivery. In this regard, Van der Ham et al. investigated the results of treatment of women with PPROM between 34 and 37 gestational weeks. 100 patients underwent the induction of labor and in 95 patients, expectant management was used. The results of this study showed that the neonatal sepsis risk does not decrease with induction of labor at $34 \mathrm{w}$ (33). In the study conducted by Neerhof et al., the best time for delivery was shown to be during the weeks 32 to 36 in women with PPROM. In this study, 34 weeks was suggested as a turning point in these patients for induction of labor; since induction of labor at this age can lower newborn hospital stay. In this retrospective study, 126 patients in expectant management and 115 patients in the induction of labor group were examined. The findings showed that the incidence of Chorioamnionitis was significantly higher in induction of labor group ( $4.8 \%$ vs. 0.9
$\%)$. Induction of labor only in 34th week raised the occurrence of hyperbilirubinemia (34). However, in the above-mentioned study, the comparison of 34 weeks with time closer to term was not mentioned. In fact, our review of the consequences of induction of labor at 34 and 36 gestational weeks in women with PPROM was one of the few studies. Kayem et al. investigated the maternal and neonatal outcomes in induction of labor and expectant management in women oxygen therapy in newborns. There was no neonatal mortality. Based on the results of this study, it might be concluded that induction of labor in 34th gestational week can increase neonatal morbidity; while, the expectant management can increase risk of infection (Chorioamnionitis) (13). As it is seen, the findings of above study somewhat confirm our findings on adverse neonatal outcomes in induction of labor at 34 weeks compared to 36 weeks. Lieman et al., in their study, tried to find the right time for induction of labor in women with PPROM. Consequently, the results of delivery before 37 weeks in 430 patients were reviewed. Accordingly, it was shown that induction of labor at 33 weeks increases morbidity significantly compared to delivery at 36 weeks. In addition, minor neonatal morbidity in the 


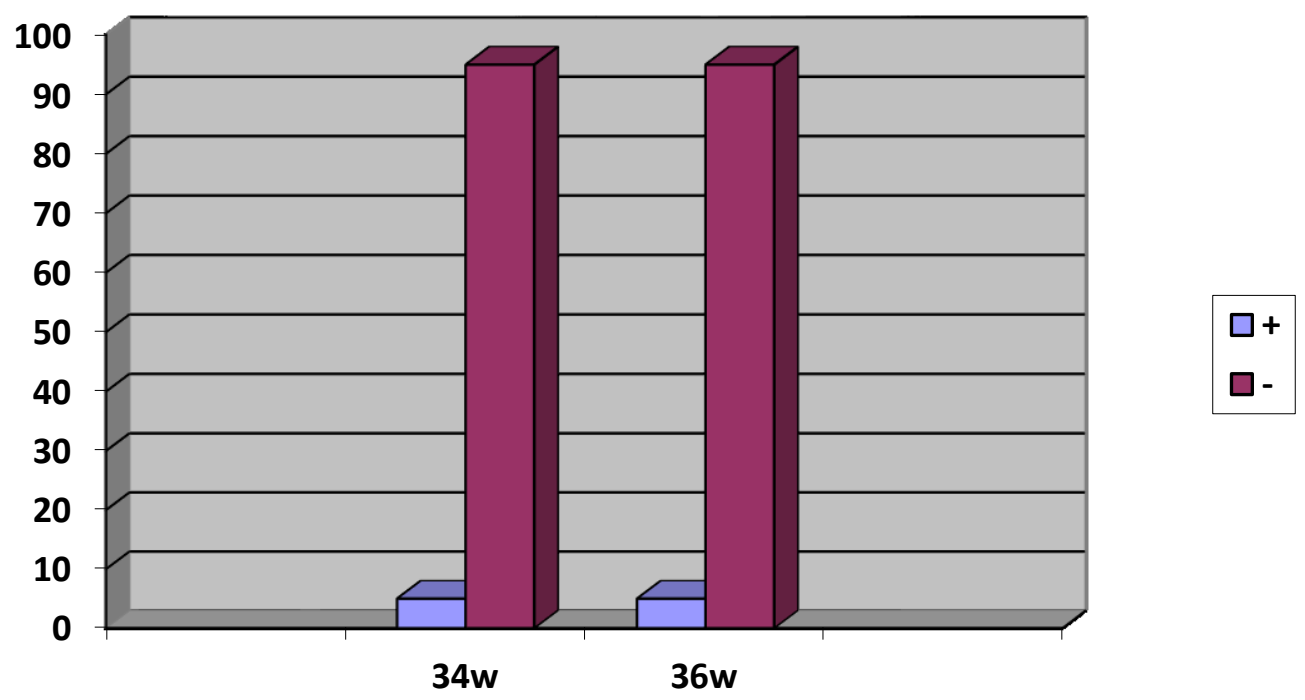

Figure 5: Frequency of Neonatal respiratory distress syndrome (+) in both group

group delivered at 34th week was significantly more common than delivery at 36th week. In addition, the average maternal and neonatal hospital stay was significantly higher in this group. It was concluded that $34 \mathrm{w}$ expectant management after 34th week did not have the same benefits (35). In this study, some findings such as increased morbidity in pregnant women at 34 weeks are consistent with the results of the current study. NICU with induction of labor at 34 weeks was significantly higher than delivered in 36 weeks.

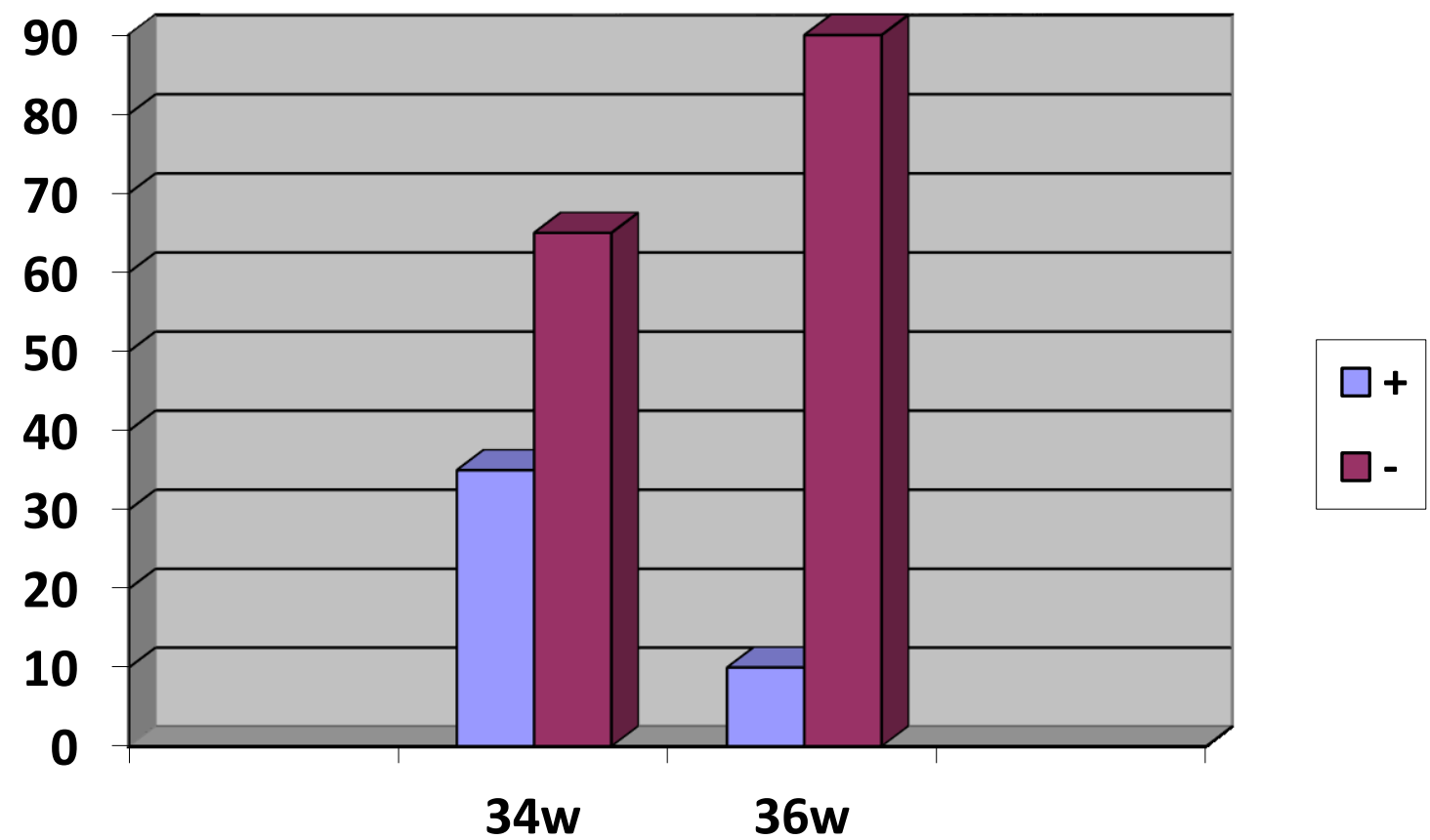

Figure 6: Frequency of neonatal NICU admission (+) in both groups Discussion 
Finally, it should be noted that the current study showed that in comparison of the outcomes of labor at 34 and 36 gestational weeks in pregnant women with PPROM, due to the increased percentage of newborns with low birth weight and increase the frequency of neonatal NICU admission in this group, termination of pregnancy at 36th week 36 is preferred. The notable maternal findings in pregnancies with PPROM terminated at 34 weeks included the need for cesarean in $10 \%$ of the patients and incidence of Chorioamnionitis in $5 \%$ of the patients. The notable maternal findings in pregnancies with PPROM terminated at 36 weeks included incidence of Chorioamnionitis in $5 \%$ of the patients. The maternal findings in pregnancies with PPROM terminated at 34 and 36 weeks had no significant difference. The neonatal findings in pregnancies with PPROM terminated at 34 weeks included birth weight between 1500 to $2500 \mathrm{~g}$ at $90 \%$, in 2500 to $3500 \mathrm{~g}$ in $5 \%$, and 3500 to $4500 \mathrm{~g}$ in 5\%; Apgar score of 3 to 5 in
$5 \%, 5$ to 7 in $30 \%$ and 7 to 10 in 65\%; neonatal respiratory distress syndrome in $5 \%$, meconium-stained amniotic fluid in $10 \%$ and NEC admission in 65\%, respectively. The neonatal findings in pregnancies with PPROM terminated at 36 weeks included birth weight between 1500 to $2500 \mathrm{~g}$ at $5 \%$, in 2500 to 3500 $\mathrm{g}$ in 95\%; Apgar score of 3 to 5 in 5\%, 5 to 7 in 5 $\%$ and 7 to 10 in 90\%; neonatal respiratory distress syndrome in 5\% and NEC admission in $10 \%$, respectively. In comparison of neonatal findings, in pregnancies with PPROM terminated at 34 and 36 weeks of gestation did not show statistically significant differences between two groups in birth weight and NICU admission. The results of the present study revealed that termination of pregnancy at 36 weeks compared to 34 weeks in pregnant women with PPROM is preferred in terms of neonatal outcomes and it is recommended; also, there might be no preference in terms of maternal outcomes.

\section{References}

1. Dagklis T, Petousis S, Margioula-Siarkou C, Mavromatidis G, Kalogiannidis I, Prapas N, et al. (2013). Parameters affecting latency period in PPROM cases: a 10-year experience of a single institution. J Matern Fetal Neonatal Med, 26(14), 1455-1458.

2. Melamed N, Hadar E, Ben-Haroush A, Kaplan B, Yogev Y. (2009). Factors affecting the duration of the latency period in preterm premature rupture of membranes. J Matern Fetal Neonatal Med, 22(11), 1051-1056.

3. Aziz N, Cheng YW, Caughey AB. (2008). Factors and outcomes associated with longer latency in preterm premature rupture of membranes. J Matern Fetal Neonatal Med, 21(11), 821-825.

4. Smith G, Rafuse C, Anand N, Brennan B, Connors G, Crane J, et al. (2005). Prevalence, management, and outcomes of preterm prelabour rupture of the membranes of women in Canada. J Obstet Gynaecol Can, 27(6), 547-553.

5. Khashoggi TY. (2004). Outcome of pregnancies with preterm premature rupture of membranes. Saudi Med J, 25(12), 1957-1961.

6. Oboro VO, Adekanle BA, Apantaku BD, Onadipe OA. (2006). Pre-term pre-labour rupture of membranes: effect of chorioamnionitis on overall neonatal outcome. J Obstet Gynaecol, 26(8), 740-743.

7. Nayot D, Penava D, Da Silva O, Richardson BS, de Vrijer B. (2012). Neonatal outcomes are associated with latency after preterm premature rupture of membranes. J Perinatol, 32(12), 970-977.

8. Simhan HN, Canavan TP. (2005). Preterm premature rupture of membranes: diagnosis, evaluation and management strategies. BJOG, 112 Suppl 132-137. 
9. Buchanan SL, Crowther CA, Levett KM, Middleton P, Morris J. (2010). Planned early birth versus expectant management for women with preterm prelabour rupture of membranes prior to 37 weeks' gestation for improving pregnancy outcome. Cochrane Database Syst Rev, (3), CD004735.

10. Manuck TA, Maclean CC, Silver RM, Varner MW. (2009). Preterm premature rupture of membranes: does the duration of latency influence perinatal outcomes? Am J Obstet Gynecol, 201(4), 414 e411-416.

11. Azria E, Anselem O, Schmitz T, Tsatsaris V, Senat MV, Goffinet F. (2012). Comparison of perinatal outcome after pre-viable preterm prelabour rupture of membranes in two centres with different rates of termination of pregnancy. BJOG, 119(4), 449-457.

12. Naef RW, 3rd, Allbert JR, Ross EL, Weber BM, Martin RW, Morrison JC. (1998). Premature rupture of membranes at 34 to 37 weeks' gestation: aggressive versus conservative management. Am J Obstet Gynecol, 178(1 Pt 1), 126-130.

13. Ames ED, Conjalka MS, Goldberg AF, Hirschman R, Jain S, Distenfeld A, et al. (1991). Hodgkin's disease and AIDS. Twenty-three new cases and a review of the literature. Hematol Oncol Clin North Am, 5(2), 343-356.

14. Mercer BM. (1998). Management of preterm premature rupture of the membranes. Clin Obstet Gynecol, 41(4), 870-882.

15. Marcellin L, Goffinet F. (2012). Are biological markers relevant for the diagnosis and the prognosis of preterm premature rupture of membranes (PPROM)? Clin Chem Lab Med, 50(6), 1015-1019.

16. Leeman LM. (1996). Premature rupture of membranes in the second trimester. J Fam Pract, 42(3), 293299.

17. Hannah ME, Hodnett ED, Willan A, Foster GA, Di Cecco R, Helewa M. (2000). Prelabor rupture of the membranes at term: expectant management at home or in hospital? The TermPROM Study Group. Obstet Gynecol, 96(4), 533-538.

18. Malinowski W. (2011). [Premature rupture of membranes one fetus from a multiple pregnancy]. Ginekol Pol, 82(10), 775-780.

19. Clark EA, Varner M. (2011). Impact of preterm PROM and its complications on long-term infant outcomes. Clin Obstet Gynecol, 54(2), 358-369.

20. Strevens H, Allen K, Thornton JG. (2010). Management of premature prelabor rupture of the membranes. Ann N Y Acad Sci, 1205123-129.

21. Locatelli A, Consonni S, Ghidini A. (2015). Preterm Labor: Approach to Decreasing Complications of Prematurity. Obstet Gynecol Clin North Am, 42(2), 255-274.

22. Lannon SM, Vanderhoeven JP, Eschenbach DA, Gravett MG, Adams Waldorf KM. (2014). Synergy and interactions among biological pathways leading to preterm premature rupture of membranes. Reprod Sci, 21(10), 1215-1227.

23. Arora KS, Miller ES. (2014). A moving line in the sand: a review of obstetric management surrounding periviability. Obstet Gynecol Surv, 69(6), 359-368.

24. Mackeen AD, Seibel-Seamon J, Muhammad J, Baxter JK, Berghella V. (2014). Tocolytics for preterm premature rupture of membranes. Cochrane Database Syst Rev, 2CD007062.

25. Mercer BM. (2003). Preterm premature rupture of the membranes. Obstet Gynecol, 101(1), 178-193.

26. Ramsey PS, Nuthalapaty FS, Lu G, Ramin S, Nuthalapaty ES, Ramin KD. (2004). Contemporary management of preterm premature rupture of membranes (PPROM): a survey of maternal-fetal medicine providers. Am J Obstet Gynecol, 191(4), 1497-1502.

27. Buchanan S, Crowther C, Morris J. (2004). Preterm prelabour rupture of the membranes: a survey of current practice. Aust N Z J Obstet Gynaecol, 44(5), 400-403.

28. Mercer BM, Crocker LG, Boe NM, Sibai BM. (1993). Induction versus expectant management in premature rupture of the membranes with mature amniotic fluid at 32 to 36 weeks: a randomized trial. Am J Obstet Gynecol, 169(4), 775-782.

29. Spinnato JA, Shaver DC, Bray EM, Lipshitz J. (1987). Preterm premature rupture of the membranes with fetal pulmonary maturity present: a prospective study. Obstet Gynecol, 69(2), 196-201. 
30. Cox SM, Leveno KJ. (1995). Intentional delivery versus expectant management with preterm ruptured membranes at 30-34 weeks' gestation. Obstet Gynecol, 86(6), 875-879.

31. Hartling L, Chari R, Friesen C, Vandermeer B, Lacaze-Masmonteil T. (2006). A systematic review of intentional delivery in women with preterm prelabor rupture of membranes. J Matern Fetal Neonatal Med, 19(3), 177-187.

32. Hannah ME, Ohlsson A, Farine D, Hewson SA, Hodnett ED, Myhr TL, et al. (1996). Induction of labor compared with expectant management for prelabor rupture of the membranes at term. TERMPROM Study Group. N Engl J Med, 334(16), 1005-1010.

33. van der Ham DP, van der Heyden JL, Opmeer BC, Mulder AL, Moonen RM, van Beek JH, et al. (2012). Management of late-preterm premature rupture of membranes: the PPROMEXIL-2 trial. Am J Obstet Gynecol, 207(4), 276 e271-210.

34. Neerhof MG, Cravello C, Haney El, Silver RK. (1999). Timing of labor induction after premature rupture of membranes between 32 and 36 weeks' gestation. Am J Obstet Gynecol, 180(2 Pt 1), 349-352.

35. Lieman JM, Brumfield CG, Carlo W, Ramsey PS. (2005). Preterm premature rupture of membranes: is there an optimal gestational age for delivery? Obstet Gynecol, 105(1), 12-17.

36. Magriples U, Crichton R, Ehrenkranz R, Copel JA. (2003). Delivery at 34 weeks is more costly than at 35 weeks in pregnancies with premature rupture of membranes. J Matern Fetal Neonatal Med, 14(1), 2225. 